Sharif University of Technology
Scientia Iranica
SCIENTIA
I RAN I CA
http://scientiairanica.sharif.edu

\title{
An endochronic-based approach to simulating pore water pressure variation during liquefaction of sand
}

\author{
A. Pak* and M.A. Iranmanesh \\ Department of Civil Engineering, Sharif University of Technology, Tehran, Iran.
}

Received 19 January 2017; received in revised form 17 December 2017; accepted 2 July 2018

\section{KEYWORDS}

Liquefaction;

Endochronic model;

Numerical simulation;

Constitutive laws;

Centrifuge test.

\begin{abstract}
Liquefaction is one of the consequences of earthquake in soil layers comprised of saturated loose sands. Various aspects of liquefaction have been investigated using different methods of field, laboratory, and numerical studies. The numerical simulation of liquefaction constitutes a major part of these research studies. The application of a proper constitutive law in numerical analysis is crucial for modeling the complicated undrained behavior of saturated sands during dynamic loading. The simplicity of formulation, the limited number of parameters, and good performance are the main features of a proper constitutive law. In this study, an endochronic-based model for simulating liquefaction of sand is implemented into the finite element program, PISA. Coupled dynamic field equations of Biot's theory with the u-p formulation are used to determine pore fluid and soil skeleton responses. Generalized Newmark method is employed for integration in time. The developed code is capable of predicting the magnitude of the generated pore water pressure, coupled with advanced constitutive laws, such as critical state two-surface plasticity, and simple models, such as Mohr-Coulomb. The simulation of cyclic simple shear and centrifuge tests using the endochronic model showed the favorable performance of this model in predicting the variation of pore water pressure in saturated soil layers subjected to earthquake excitations.
\end{abstract}

(C) 2020 Sharif University of Technology. All rights reserved.

\section{Introduction}

The process of pore pressure buildup associated with the reduction of shearing resistance is relatively frequent in loose saturated sandy soils during an earthquake. The destructive effect of the earthquakeinduced liquefaction on buildings and infrastructural systems is well recognized. Numerical models capable of predicting liquefaction phenomenon can be useful

*. Corresponding author. Tel.: +98 2166164225 ; E-mail addresses: pak@sharif.edu (A.Pak); iranmanesh@mehr.sharif.edu (M.A.Iranmanesh)

doi: $10.24200 /$ sci. 2018.20639 for providing the means to mitigate its hazard. In the numerical modeling of liquefaction, it is necessary to consider the solid-pore fluid interaction. In addition, employing an appropriate constitutive law for simulating sand behavior under complex loading conditions is very important. Numerical models require constitutive laws that predict the stress-strain relationship, the soil stiffness degradation, and the changes in the pore water pressure [1]. Numerical modeling of soil liquefaction using advanced constitutive laws and numerical techniques has been the subject of many investigations in recent years. Bounding surface models, nested surface models, generalized plasticity models, and micromechanical and empirical models are examples of constitutive laws for predicting the 
behavior of saturated sand under dynamic loading, especially liquefaction phenomenon [2]. In this study, the capability of an endochronic-based approach to the evaluation of the possibility of flow liquefaction is investigated.

Endochronic theory was first introduced by Valanis [3] to describe the nonlinear material response and reduce the difficulty of the classical theory of plasticity for metals [4]. Valanis and Read then extended this theory for soils [5], and also Bazant and Krizek developed an entirely new type of the constitutive law based on this theory for the liquefaction of sand [6]. They represented the densification or volumetric strains caused by cyclic shearing in terms of endochronic variables. Moreover, Zienkiewicz et al. used endochronic variables to describe the volumetric strains caused by cyclic loading [7]. In both of cases, the volumetric strains were then incorporated into a pore water pressure relation, which gave the increment of pore water pressure in terms of the volumetric strain and the bulk moduli of the soil skeleton. The main feature of the endochronic model is the application of the intrinsic time parameter, which is an independent scalar variable in which increment generally depends on both time and deformation. However, the dependence on time increments disappears in the case of timeindependent inelastic behavior, as is the case with sand. In this case, the intrinsic time parameter represents the length of the path traced by the states of the material in strain space [6-8]. Lopez and Blazquez improved the endochronic model to simulate the behavior of undrained sand under dynamic loading [1]. Their proposed model works for both small and large strains and incorporates contractive and dilative properties of the sand into a unified numerical scheme. These features allow for predicting the liquefaction phenomenon correctly for different initial relative densities of the soil.

The proper simulation of pore pressure variation due to contractive or dilative behavior of saturated sands is a major part of liquefaction analysis. The main objective of this paper is to predict pore pressure variation by using stress and strain fields history obtained by employing a constitutive law such as MohrCoulomb. In this study, the endochronic formulation is only used to simulate the variation of pore water pressure and predict the possibility of the occurrence of liquefaction based on the stress/strain fields calculated by any other constitutive model. This is a useful and efficient ability to forecast the occurrence of liquefaction by using simple constitutive laws such as MohrCoulomb, provided that they are combined with the endochronic formulation. In the next sections, after a brief description of the endochronic formulations, its validity is evaluated by experimental data of cyclic simple shear and centrifuge tests.

\section{A brief description of the endochronic formulation}

\subsection{Contractive behavior}

The densification of sand results from the irreversible rearrangement of configurations of sand grains associated with the application of a deviatoric strain. It is appropriate to define this cumulative rearrangement of grain configurations by a suitable parameter, $\xi$, called "rearrangement measure", which is a function of the deviatoric component of the strain tensor [1]. In the mathematical form, we have:

$$
\xi=\int \mathrm{d} \xi=\int|\mathrm{d} \gamma|,
$$

where $\gamma=\left(8 J_{2}\left(\varepsilon_{i i}\right)\right)^{1 / 2}$, and $J_{2}(\ldots)$ denotes the second invariant of the tensor in parenthesis $[1,9]$. Another parameter, $\zeta$, called "densification measure" can be defined as a function of the deviatoric strain:

$$
\zeta=\int \mathrm{d} \zeta=\int \frac{n}{4}|100 \gamma|^{(n-1)}|\mathrm{d} \gamma|
$$

We have the densification law in the incremental form as follows:

$$
\mathrm{d} \varepsilon_{v}=-\frac{\mathrm{d} \zeta}{1+\alpha \zeta}=-\frac{n}{4} \frac{|100 \gamma|^{(n-1)}}{1+\alpha \zeta}|\mathrm{d} \gamma|,
$$

where $\alpha$ and $n$ are the two parameters of the densification law, and $\gamma=\gamma_{\max } \sin \omega t$ is a cyclic function with amplitude $\gamma_{\max }$. By integrating Eqs. (1), (2) and (3), we have:

$$
\begin{aligned}
& \xi=4 N \gamma_{\max }, \\
& \zeta=N \gamma_{\max }^{n}, \\
& \varepsilon_{v}=-\frac{1}{\alpha} \ln (1+\alpha \zeta),
\end{aligned}
$$

where $N$ is the number of cycles of shear strain loading [9]. In the case of a non-harmonic input motion, regardless of the type of loading, in each time step the values of $N_{e q}$ (equivalent number of cycles), $\gamma_{\max , e q}$ (equivalent maximum shear strain), and $n$ are obtained by solving the set of Eqs. (4), (5) and (7).

The parameters $\alpha$ and $n$ are considered to be dependent only on the initial relative density.

$$
\begin{aligned}
& n=A \ln (N)+B, \\
& \alpha=C N^{-D},
\end{aligned}
$$

where $A, B, C$, and $D$ are defined as follows:

$$
A=\frac{1}{2} \frac{e_{\max }^{2}-e_{\min }^{2}}{1+e_{\max }-D r_{0}\left(e_{\max }-e_{\min }\right)},
$$




$$
\begin{aligned}
& D=\frac{1}{2}\left(e_{\max }^{2}-e_{\min }^{2}\right), \\
& B=D+1,
\end{aligned}
$$

where $e_{\max }$ and $e_{\min }$ are the maximum and minimum void ratios, respectively, and $D r_{0}$ is the initial relative density of the soil. Variable $C$ is a model parameter that must be determined experimentally from test data. It is the value of $\alpha$ in the first cycle and depends on the type of sand and the initial relative density [9].

\subsection{Calculation of elastic and plastic response} If the deviatoric components of the stress $(\tau)$ and the strain $(\gamma)$, are only considered, the total strain increment in each time step can be written as follows:

$$
\begin{aligned}
& \mathrm{d} \gamma=\mathrm{d} \gamma^{e}+\mathrm{d} \gamma^{p}, \\
& \mathrm{~d} \gamma^{e}=\frac{\mathrm{d} \tau}{G}, \\
& \mathrm{~d} \gamma^{p}=\frac{\tau|\mathrm{d} \gamma|}{G \tau_{D}\left(1+\frac{\alpha^{\prime}}{\beta} \xi\right)^{\beta}}=\frac{\tau \operatorname{sign}(\mathrm{d} \gamma) \mathrm{d} \gamma}{G \tau_{D}\left(1+\frac{\alpha^{\prime}}{\beta} \xi\right)^{\beta}},
\end{aligned}
$$

where $\mathrm{d} \gamma^{e}$ and $\mathrm{d} \gamma^{p}$ are elastic and plastic parts of the deviatoric component of strain, respectively. $\alpha^{\prime}, \beta$, and $\tau_{D}$ are the parameters of the flow law. The value of these parameters for all types of sands are found to be constant $\left(\alpha^{\prime}=1, \beta=0.7, \tau_{D}=0.0005\right)$. Note that these parameters are not model parameters [1]. Detailed information about these parameters and their values is available in [10]. In order to take into account the reduction of the tangent elastic shear modulus due to the loss of the stiffness of the soil under cyclic loading, a general formula proposed by Papadimitriou et al. [11] has been used as follows:

$$
G=\frac{G_{\max }}{T}=\frac{1}{T} \frac{B_{\mathrm{g}} p_{\mathrm{a}}}{0.3+0.7 e^{2}}\left(\frac{p}{p_{\mathrm{a}}}\right)^{0.5}
$$

where $B_{\mathrm{g}}$ is the model parameter, which depends on the type of sand. $p$ is the effective confining pressure, $e$ is the void ratio, and $p_{\mathrm{a}}$ is the atmospheric pressure. The degradation parameter, $T$, can be expressed as follows:

$$
\begin{aligned}
& T=1+2 C_{t}\left|\eta-\eta_{0}\right| \quad \text { (first loading), } \\
& T=1+C_{t}\left|\eta-\eta_{s r}\right| \quad \text { (unloading and reloading), }
\end{aligned}
$$

where $C_{t}$ is the tangential shear modulus parameter. $\eta$, $\eta_{0}$, and $\eta_{s r}$ are the current, initial, and at last reversal values of $\tau / \sigma_{v}^{\prime}$, respectively, where $\sigma_{v}^{\prime}$ is the current effective vertical stress $[9,11,12]$.

\subsection{Dilative behavior}

For simulating the dilative behavior of soil, a new parameter for representing the stiffness degradation of the soil from the beginning of the loading is introduced.

$$
\psi=\frac{G_{0}}{G-G_{0}},
$$

where $G_{0}$ and $G$ are the initial and current elastic tangent shear moduli, respectively. At the beginning of the loading, when $G=G_{0}$, the parameter $\psi$ takes the value of infinity and has the value of unity when $G$ approaches zero. In the numerical implementation, in order to prevent instability, the value of $\psi$ must be considered as a large number at the beginning. The dilatancy, which relates the plastic volumetric strains and the plastic shear strains, is defined as a function of the degradation parameter, $\psi$, and another model parameter, $\alpha_{p}$ :

$$
\mathrm{d} \varepsilon_{v}^{p}=\left(1+\alpha_{p}\right) \psi\left|\mathrm{d} \gamma^{p}\right| .
$$

The parameter $\alpha_{p}$ is a function of the type of sand, the initial relative density, and the Cyclic Stress Ratio (CSR) $[9,13]$.

\subsection{Calculation of pore water pressure increment}

The excess pore water pressure increment in the undrained sand, $\mathrm{d} p_{w}$, is as follows:

$$
\mathrm{d} p_{w}=-M_{d} \mathrm{~d} \varepsilon_{v}
$$

where $\mathrm{d} \varepsilon_{v}$ is the increment of volumetric strain that is calculated from Eqs. (3) and (18) for contractive and dilative behaviors of sand, respectively. $M_{d}$ is the constrained modulus of the drained sand and usually relates to the initial and current values of effective stresses as follows:

$$
M_{d}=\frac{K_{d}}{\left(\sigma_{v 0}^{\prime}\right)^{n^{*}}}\left(\sigma_{v}^{\prime}\right)^{0.5},
$$

where $K_{d}$ and $n^{*}$ are the constrained modulus parameters that are dependent on the type of sand, and $\sigma_{v 0}^{\prime}$ is the initial effective vertical stress [1].

\subsection{Effect of changes of the internal structure of the soil}

In order to take into account the effect of changes of the internal soil structure after dilation, Eq. (3) should be modified as follows:

$$
\mathrm{d} \varepsilon_{v}=-I_{s}\left(\frac{n}{4}\right) \frac{|100 \gamma|^{(n-1)}}{1+\alpha \gamma}|\mathrm{d} \gamma|,
$$

where $I_{s}$ is another model parameter that quantifies the degree of change of the internal soil structure in dilation [1]. 
Table 1. Endochronic parameters for Nevada sand [1,9].

\begin{tabular}{cc}
\hline Parameter & Value \\
\hline$C$ & $50 D r_{0}+5$ \\
$n^{*}$ & 0.425 \\
$K_{d}$ & $449 /(C S R)^{1.8}$ \\
$C_{t}$ & 1.6 \\
$B_{g}$ & 140 \\
$I_{s}$ & $0.51 \exp (11.65 C S R)$ \\
$\alpha_{p}$ & -0.85 \\
\hline
\end{tabular}

\subsection{Model parameters}

This model contains seven parameters. $C$ is a parameter of densification law that depends on the initial relative density and type of the sand [10]. Drained constrained modulus parameters $n^{*}$ and $K_{d}$ are dependent on the type of the sand. While $n^{*}$ is constant for each sand, $K_{d}$ depends on CSR. $C_{t}$ and $B_{g}$ are tangent shear modulus parameters. Finally, $I_{s}$ and $\alpha_{p}$ are the dilation parameters. The manner of calibration of these parameters by using cyclic simple shear test data is beyond the scope of this paper and is available in the study of Blazquez and Lopez [9]. These parameters for Nevada sand are presented in Table 1.

\section{Formulation of the FEM model}

The performance of the endochronic model is evaluated using the geotechnical finite element code PISA which has been modified for fully-coupled dynamic nonlinear effective stress analysis of saturated porous media. The first version of the finite element program PISA was developed in the University of Alberta with the early name of SAGE [14]. Later on, a commercial version of this program was released under the name of PISA [15]. This geotechnical software is used to analyze stress and deformation fields in plane strain, plane stress, and axisymmetric problems. Drained and undrained analyses can be performed using a wide range of elastic, elastoplastic, and critical state models. Pak [16] and Shahir [17] further increased the capabilities of this program by completing the formulation to simulate THM (Thermo Hydro Mechanical) and dynamic problems, respectively. In addition, the finite element program, PISA, was modified to include the capability of considering variations of permeability coefficient during liquefaction $[18,19]$. In this program, saturated soil is simulated as a two-phase material based on Biot's theory [20] of consolidation. The u$\mathrm{p}$ formulation is used in the mentioned code, where the displacement of the soil skeleton, $\mathrm{u}$, and pore pressure, $\mathrm{p}$, are the primary unknowns. The $\mathrm{u}-\mathrm{p}$ formulation is defined by the equation of motion for the solid-fluid mixture and the equation of mass conservation for the fluid phase, incorporating the equation of motion in the fluid phase and Darcys' law. By applying the finite element method for spatial discretization, the $u-p$ formulation is as follows [21]:

$$
\begin{aligned}
& \mathbf{M} \ddot{\mathbf{U}}+\int_{v} \mathbf{B}^{T} \boldsymbol{\sigma}^{\prime \prime} \mathrm{d} V-\mathbf{Q P}-\mathbf{f}^{(s)}=0, \\
& \mathbf{Q}^{T} \dot{\mathbf{U}}+\mathbf{H P}-\mathbf{S} \dot{\mathbf{P}}-\mathbf{f}^{(p)}=0
\end{aligned}
$$

where $\mathbf{M}$ is the mass matrix, $\mathbf{U}$ is the displacement vector, $\mathbf{B}$ is the strain-displacement matrix, $\boldsymbol{\sigma}^{\prime \prime}$ is the effective stress vector, $\mathbf{Q}$ is the discrete gradient operator coupling the solid and fluid phases, $\mathbf{P}$ is the pore pressure vector, $\mathbf{S}$ is the permeability matrix, and $\mathbf{H}$ is the compressibility matrix. The vectors $\mathbf{f}^{(s)}$ and $\mathbf{f}^{(p)}$ represent the effect of body forces and prescribed boundary conditions for the solid-fluid mixture and the fluid phase, respectively. In Eq. (22) (equation of motion), the first term represents inertia force of the mixture, followed by internal force due to soil skeleton deformation, and that due to pore-fluid pressure. In Eq. (23) (mass conservation), the first two terms represent the rate of the volume change for the soil skeleton and the fluid phases, respectively, followed by the seepage rate of the pore fluid. To complete the numerical solution, it is necessary to integrate the above equations into time. Here, the generalized Newmark method is used [22].

\section{Variation of the permeability coefficient during liquefaction}

It has been reported in a number of investigations that one of the main mechanisms causing substantial settlement is the significant increase of the soil permeability during seismic excitation. Using the Kozeny-Carman equation, Arulanandan and Sybico [23] concluded that a change in the permeability coefficient during liquefaction was due to the change in pore shape factor and tortuosity. This change takes place because of the reduction of contacts among sand particles. Jafarzadeh and Yanagisawa conducted a number of unidirectional shaking table tests to study the settlement of the dry and saturated sand columns in various conditions. They observed that the average permeability coefficient was 5-6 times greater during seismic excitation than its static value [24]. Numerical studies by Rahmani et al. [18] and Shahir et al. [19] showed that the numerical analysis of liquefaction using the initial value of the permeability coefficient caused the settlement to be predicted smaller than the measured soil settlement.

In this study a variable permeability function proposed by Shahir et al. [19] is used. In this function, a direct relationship between the permeability coefficient and the excess pore water pressure ratio is 


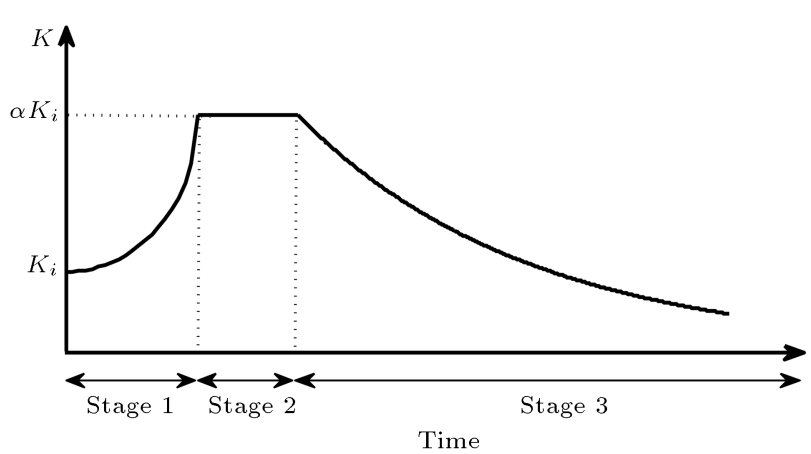

Figure 1. Schematic view of the proposed permeability function. Stage 1: Pore water pressure buildup phase; Stage 2: Liquefied state; stage 3: Consolidation state [19]. suggested (Figure 1).

$$
\begin{aligned}
& \frac{k_{b}}{k_{i}}=1+(\alpha-1) r_{u}^{\beta_{1}} \quad \text { During PWP build-up phase } \\
& \left(r_{u}<1\right), \\
& \frac{k_{b}}{k_{i}}=\alpha \quad \text { During liqufaction } \\
& \left(r_{u}=1\right), \\
& \frac{k_{b}}{k_{i}}=1+(\alpha-1) r_{u}^{\beta_{2}} \quad \text { During consolidation phase } \\
& \left(r_{u}<1\right),
\end{aligned}
$$

where $k_{i}$ is the initial permeability coefficient, $k_{b}$ is the permeability coefficient during excitation, and $r_{u}$ (excess pore water pressure ratio) is defined as the ratio of the difference between current pore pressure and hydrostatic pore pressure over the initial effective vertical stress $\left(r_{u}=\Delta u / \sigma_{v 0}^{\prime}\right) . \quad \alpha, \beta_{1}$, and $\beta_{2}$ are positive material constants that were calibrated by Shahir et al. $[19,25]$ and are selected to be 20, 1, and 8.9 , respectively, for Neveda sand.

\section{Evaluation of the performance of the model}

The performance of the endochronic model has been evaluated by comparing its results with those of two cyclic simple shear tests on Nevada sand with two different initial void ratios [26] and those of class B prediction of the experiment no. 1 of VELACS (Verification of Liquefaction Analysis by Centrifuge Studies) project [27]. The calculations are conducted along with a simple constitutive law, Mohr-Coulomb, and an advanced one, critical state two-surface plasticity (Manzari and Dafalias, 1997) [28]. In each time step, stress and strain tensors are calculated by one of these constitutive laws; then, pore water pressure increment is calculated through endochronic formulations using stress/strain fields calculated by the mentioned constitutive laws. Therefore, the endochronic formulations are coupled with another constitutive law whose role is to calculate stress/strain fields. This is why we believe that endochronic model is coupled with Mohr-Coulomb (M-C), or endochronic model is coupled with the model of Manzari \& Dafalias (M\&D). The increment of pore water pressure calculated by the endochronic model is not exported to the constitutive laws for calculating stress and strains in each time step. Note that the endochronic model in this research is only used to simulate the variation of pore water pressure during loading stages, because the investigation of the possibility of the liquefaction occurrence is the main purpose of this work, and the variation of pore pressure has a major role in this context.

\subsection{Cyclic simple shear tests}

The test samples used for the cyclic simple shear test are the cylindrical samples with a diameter of $6.35 \mathrm{~cm}$ and a height of $2.54 \mathrm{~cm}$. These are Laboratory tests conducted on Nevada sand by the earth technology corporation [26]. In order to simulate this test, first, the soil sample is subjected to a normal stress of $160 \mathrm{kPa}$. During this stage, drainage is allowed. After that, by applying shear stress in the undrained situation, the shear strain and the effective normal stress variation and the excess pore water pressure are simulated. In the numerical model, 12 elements that are eightnode quadrilateral, plane strain-type elements have been used. Figure 2 shows the finite element mesh and boundary conditions. Stress path of this test is demonstrated in Figure 3. Material properties for the Mohr-Coulomb model consist of Young's modulus of $6000 \mathrm{kPa}$, Poison's ratio of 0.3 , and a friction angle of 33 degrees. Table 2 presents the parameters of the model of Manzari \& Dafalias for Nevada sand. Figures 4 to 7 show variations of pore water pressure during cyclic loading on the saturated sand sample obtained by the experimental records and numerical simulations at the center of the specimen. In these figures, the threshold of liquefaction occurrence $\left(r_{u}=1\right)$ is specified by a

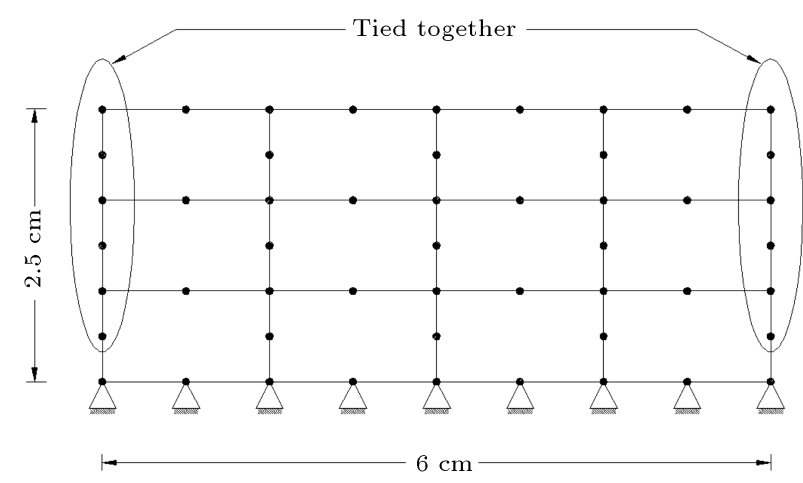

Figure 2. Finite element mesh and boundary conditions for cyclic simple shear test [26]. 
Table 2. Model parameters of Manzari \& Dafalias (1997) [28] for Nevada sand.

\begin{tabular}{ll}
\hline \multicolumn{1}{c}{ Parameter } & Value \\
\hline Elastic Parameters: & \\
$G_{0}(\mathrm{kPa})$ & 31400 \\
$K_{0}(\mathrm{kPa})$ & 31400 \\
$a$ & 0.6 \\
$C_{\text {Critical State Paremeters: }}$ & \\
$M_{c}$ & 1.14 \\
$M_{e}$ & 1.14 \\
$\lambda$ & 0.025 \\
$\left(e_{c}\right)_{\text {ref }}$ & 0.8 \\
State Paremeters: & \\
$K_{c}^{b}$ & 3.975 \\
$K_{e}^{b}$ & 2.0 \\
$K_{c}^{d}$ & 4.2 \\
$K_{e}^{d}$ & 0.07 \\
Hardening Parameters: & \\
$h_{0}$ & 800.0 \\
$m$ & 0.056 \\
Dilation Parameter: & \\
A & 0.6 \\
\hline
\end{tabular}

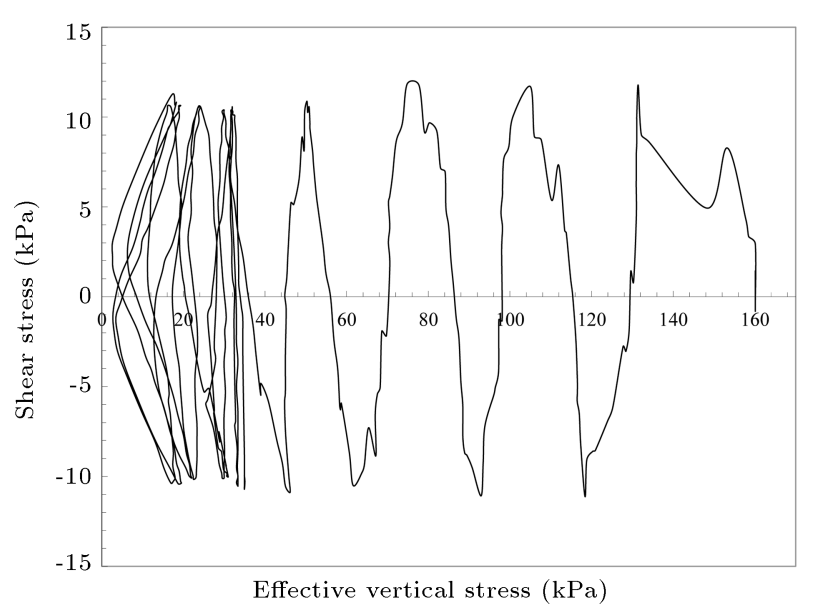

Figure 3. Stress path for cyclic simple shear test.

dashed line, and the results of the endochronic model coupled with the critical state tow-surface plasticity model proposed by Manzari \& Dafalias (1997) and the endochronic model coupled with Mohr-Coulomb for Nevada sand with two void ratios of 0.65 and 0.72 are illustrated.

Figures 4 to 7 represent the favorable performance of the endochronic formulation for simulating variations of pore water pressure in undrained cyclic simple shear tests. As can be seen, variations of pore water pressure in the simulation by the endochronic model coupled with the model of Manzari \& Dafalias (1997)

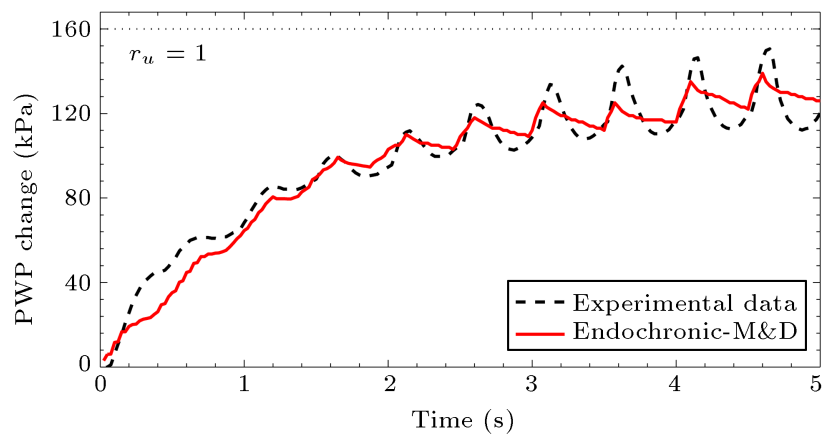

Figure 4. Variation of excess pore water pressure over time in the undrained cyclic simple shear test for Nevada sand with a void ratio of 0.65 by the endochronic model coupled with the model of Manzari \& Dafalias (1997) [26].

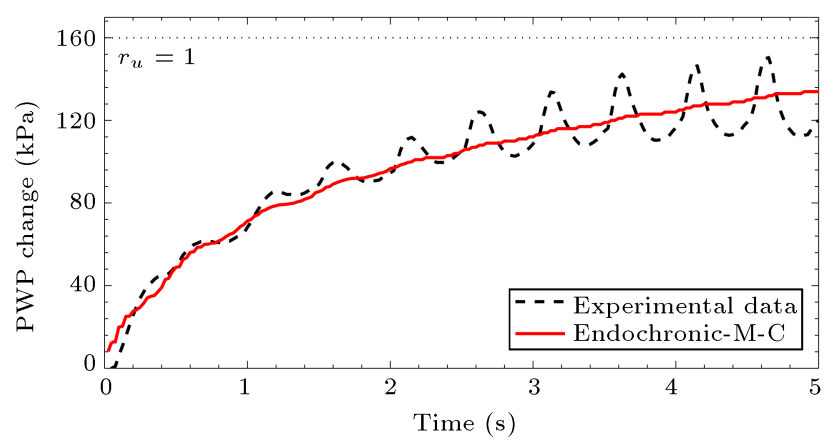

Figure 5. Variation of excess pore water pressure over time in the undrained cyclic simple shear test for Nevada sand with a void ratio of 0.65 by the endochronic model coupled with Mohr-Coulomb [26].

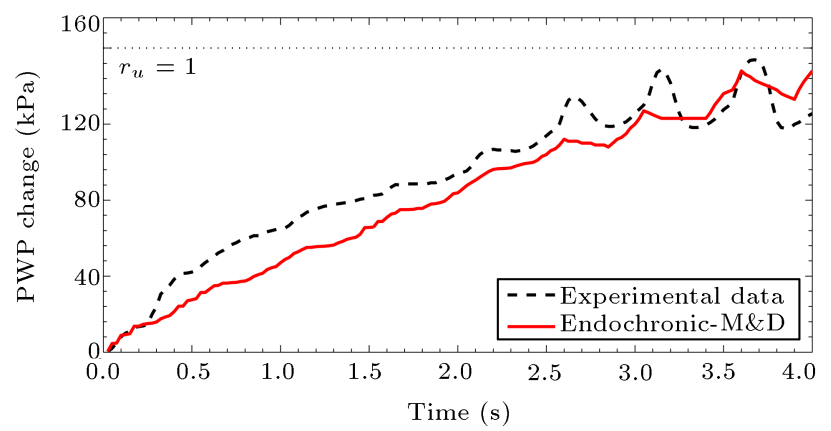

Figure 6. Variation of excess pore water pressure over time in the undrained cyclic simple shear test for Nevada sand with a void ratio of 0.72 by the endochronic model coupled with the model of Manzari \& Dafalias [26].

are in good agreement with experimental data, and also variation of pore water pressure in the simulation with endochronic model coupled with Mohr-Coulomb is acceptable.

The trend and number of oscillations in the simulation by the endochronic model coupled with the model of Manzari \& Dafalias (1997) are more compatible with experimental results than those in the simulation by the endochronic model coupled with 


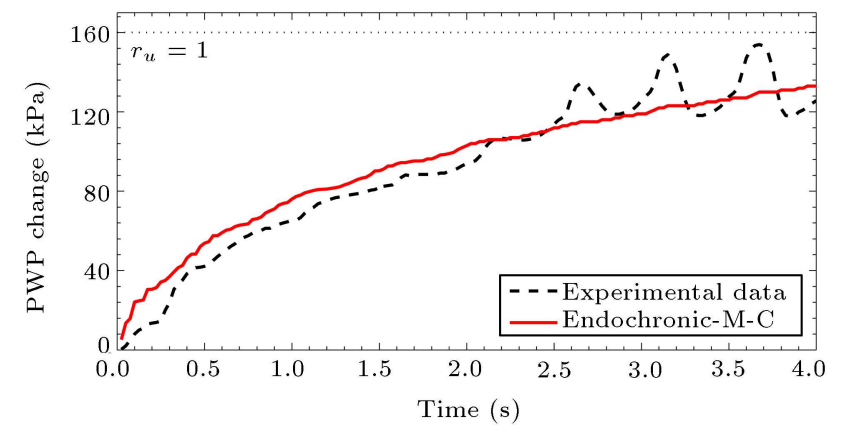

Figure 7. Variation of excess pore water pressure over time in the undrained cyclic simple shear test for Nevada sand with a void ratio of 0.72 by the endochronic model coupled with Mohr-Coulomb [26].

Mohr-Coulomb. However, by using the endochronic model coupled with Mohr-Coulomb, it is still possible to predict the onset of liquefaction.

\subsection{Centrifuge test}

In order to simulate a centrifuge test, the class B prediction of the experiment no. 1 of VELACS project is considered here [27]. The experiment no. 1 consists of a $20 \mathrm{~cm}$ high, horizontal, uniform Nevada sand layer, which is placed in a laminar box at a relative density of about 40 percent. The laminar box is made of rectangular aluminum rings stacked on top of each other with roller bearings in between. The purpose of using laminar box is to simulate the response of a semi-infinite sand layer during shaking. A sketch of the laminar box and the instrumentation used for this experiment is presented in Figure 8. In this figure, $\mathrm{P} 1$ to $\mathrm{P} 8$ indicate pore pressure transducers; AH1 to AH5 and AV1 to AV5 are accelerometers for horizontal and vertical directions, respectively, and also LVDT1 and LVDT2 are used for recording horizontal and vertical displacements. The sand layer is fully saturated with water, spun at a centrifuge acceleration of $50 \mathrm{~g}$, and excited horizontally at the base with the target prototype accelerogram reproduced in Figure 9 . A zero vertical acceleration was considered.

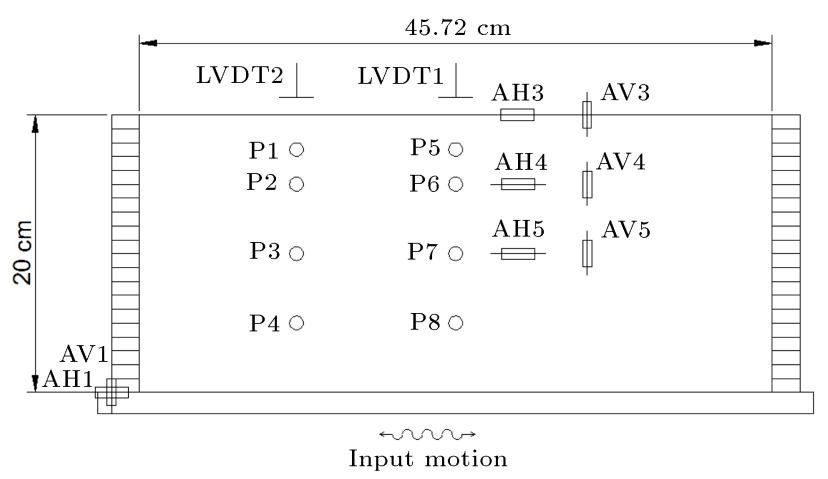

Figure 8. Cross-sectional view of the centrifuge experiment no. 1 [27].

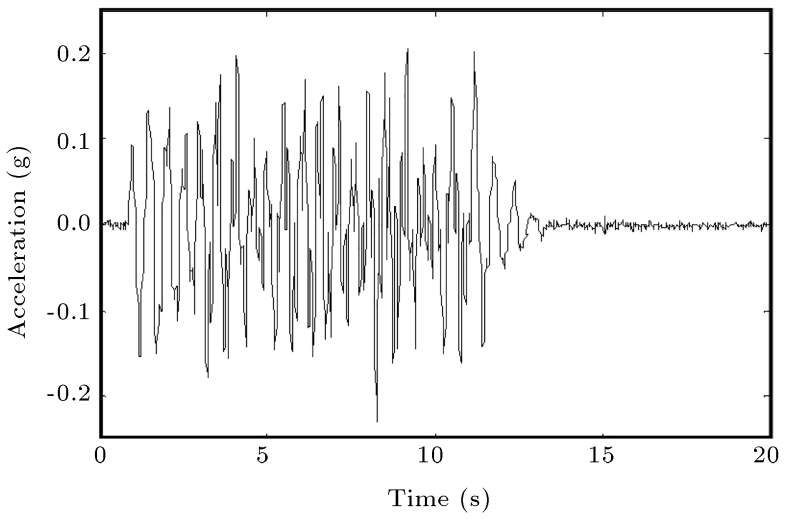

Figure 9. Horizontal input motion at the bottom of laminar box [27].

Numerical modeling is conducted on a prototype scale using a two-dimensional formulation with a planestrain condition. Herein, 64 rectangular elements with 4 nodes for pore pressures and 8 nodes for displacements are used. The mesh is regular and uniform, as shown in Figure 10. The laminar box is modeled with the constraint of laterally tied nodes. The displacements of nodes located at the two ends of the soil at the same level are restrained to have the same value. The base nodes are fixed in both horizontal and vertical directions. The dissipation of pore pressure is allowed only via the top surface of the soil; the lateral boundaries and the base are kept impermeable. The effect of the lateral inertia of the rings is neglected. Computations are performed in two steps; first, a static analysis due to the application of gravity (model's own weight) is performed before seismic excitation. The resulting fluid hydrostatic pressures and stress states along the soil column are used as the initial conditions for the subsequent dynamic analysis. Material properties and model parameters of Mohr-Coulomb and Manzari \& Dafalias were presented in the previous example.

The observed and predicted time histories of pore pressure using the endochronic formulation coupled

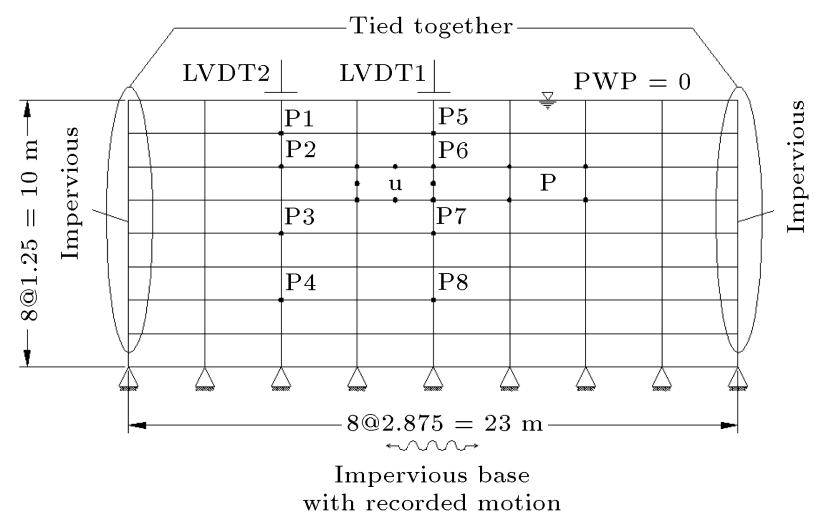

Figure 10. Finite element mesh and boundary conditions [27]. 
with Manzari \& Dafalias (1997) and Mohr-Coulomb models for transducers P5 to P8 are shown in Figures 11 to 18 . The conditions of transducers P1 to P4 are exactly the same as those of transducers P5 to P8. In these figures, the threshold of liquefaction occurrence $\left(r_{u}=1\right)$ is specified by a dashed line.

Figures 11 to 18 represent a favorable performance of the endochronic model coupled with the model of Manzari \& Dafalias (1997) and a relatively acceptable performance of the endochronic model coupled with Mohr-Coulomb for simulating the variation

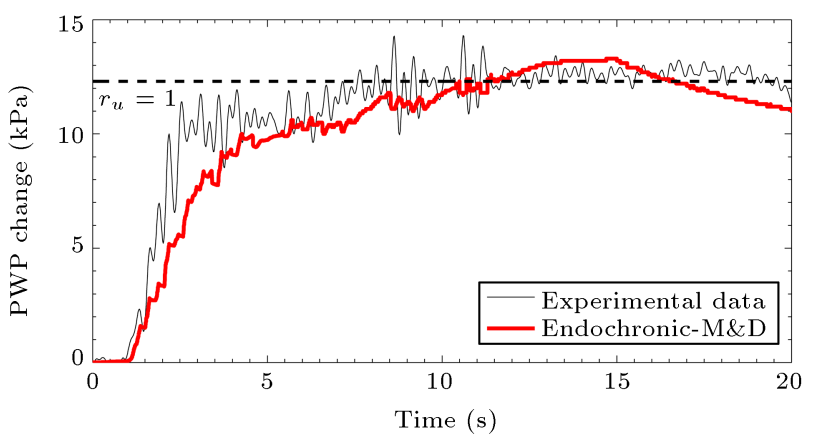

Figure 11. Observed and predicted time histories of pore pressure in transducer P5 using the endochronic model coupled with the model of Manzari \& Dafalias (1997) [27].

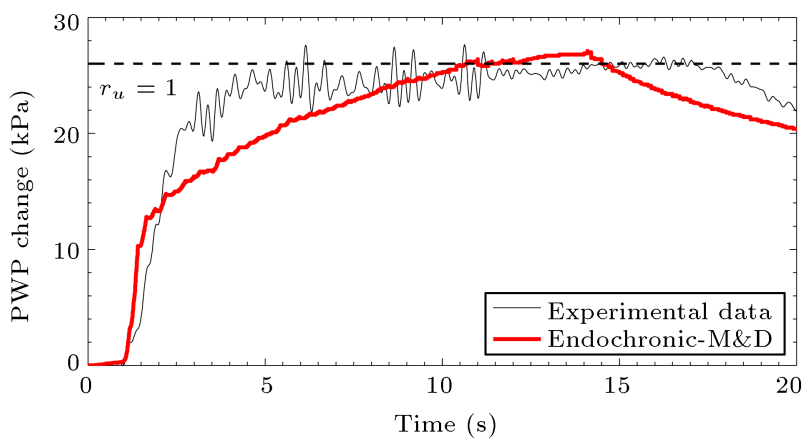

Figure 12. Observed and predicted time histories of pore pressure in transducer P6 using the endochronic model coupled with the model of Manzari \& Dafalias (1997) [27].

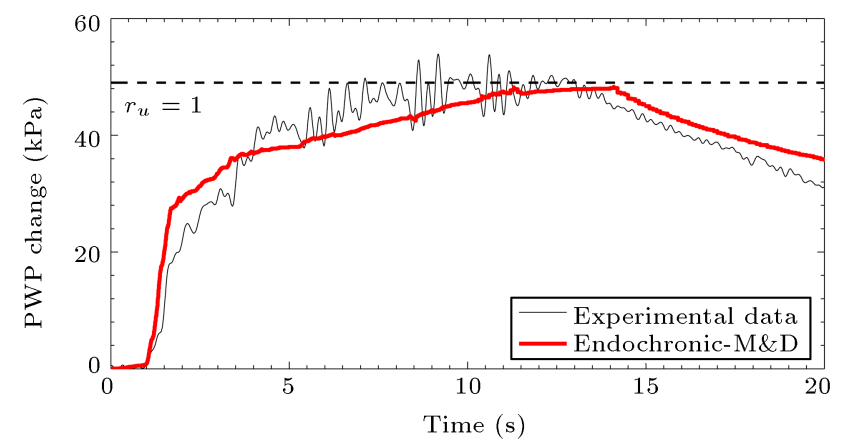

Figure 13. Observed and predicted time histories of pore pressure in transducer P7 using the endochronic model coupled with the model of Manzari \& Dafalias (1997) [27]. of pore water pressure in Model No. 1 of VELACS. Experimental results indicate that liquefaction does not occur at all depths of the sand layer at the same time. Liquefaction first takes place at the upper levels and spreads gradually to greater depths such that the sand layer liquefies at points P5 and P6 first. After that, at point $\mathrm{P} 7$, pore water pressure increases to an amount of about the normal stress, whereas the effective stress is still non-zero at point P8. As can be seen, this trend can be observed in simulation by the endochronic model coupled with both Manzari \& Dafalias (1997) and Mohr-Coulomb. Figure 19 presents

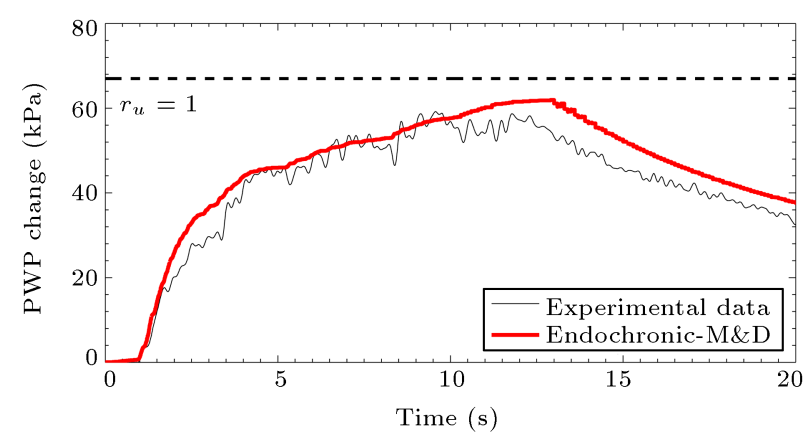

Figure 14. Observed and predicted time histories of pore pressure in transducer P8 using the endochronic model coupled with the model of Manzari \& Dafalias (1997) [27].

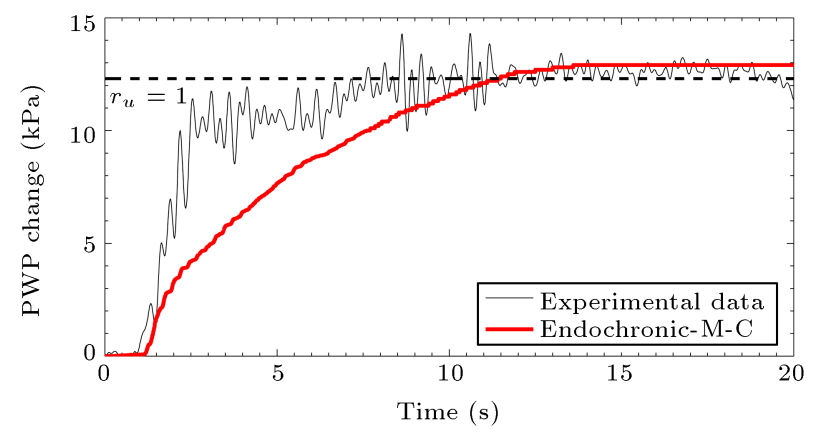

Figure 15. Observed and predicted time histories of pore pressure in transducer P5 using the endochronic model coupled with Mohr-Coulomb [27].

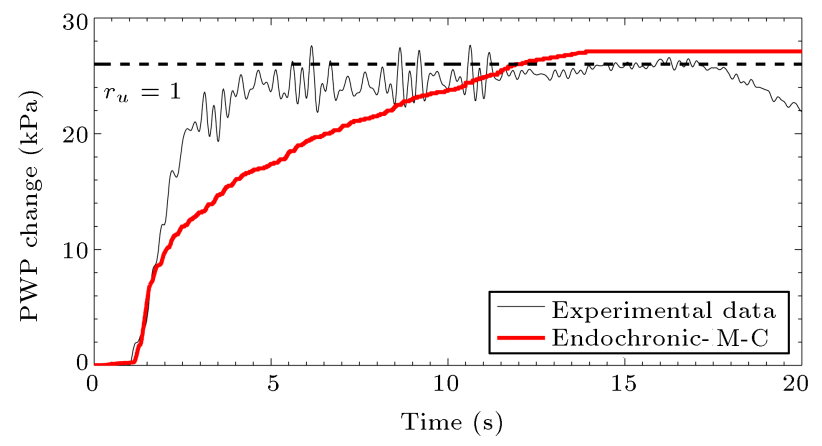

Figure 16. Observed and predicted time histories of pore pressure in transducer $\mathrm{P} 6$ using the endochronic model coupled with Mohr-Coulomb [27]. 


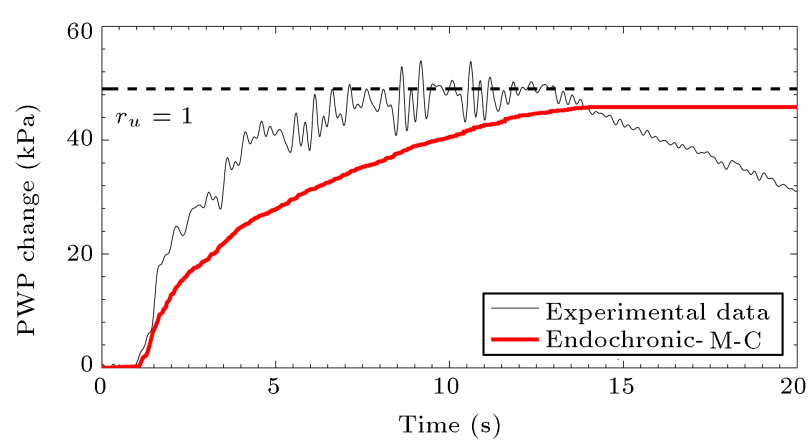

Figure 17. Observed and predicted time histories of pore pressure in transducer P7 using the endochronic model coupled with Mohr-Coulomb [27].

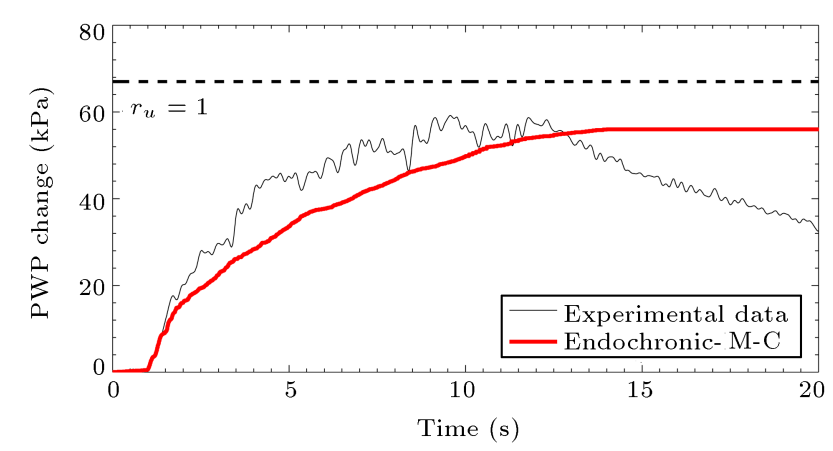

Figure 18. Observed and predicted time histories of pore pressure in transducer $\mathrm{P} 8$ using the endochronic model coupled with Mohr-Coulomb [27].

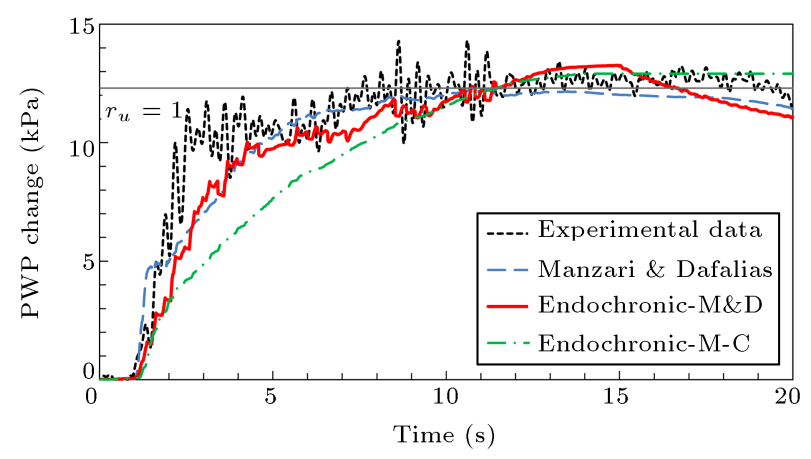

Figure 19. Comparison between predicted time histories of pore pressure in transducer P5 using the model of Manzari and Dafalias (1997), the endochronic model coupled with the model of Manzari and Dafalias, and the endochronic model coupled with Mohr-Coulomb.

a comparison between predicted time histories of pore pressure in transducer P 5 by the model of Manzari and Dafalias (1997), the endochronic model coupled with the model of Manzari \& Dafalias, and that coupled with Mohr-Coulomb. The Mohr-Coulomb model by itself is not capable of predicting the pore pressure variation during liquefaction. Moreover, the dissipation of pore water pressure and consolidation stage after vanishing the horizontal acceleration is simulated successfully with the endochronic model coupled with the model of Manzari \& Dafalias (1997). The endochronic model coupled with Mohr-Coulomb, however, cannot predict the consolidation stage, and the trend of the variation of pore water pressure does not acceptably match the experimental data. However, it can fairly predict the occurrence of liquefaction at all depths. Therefore, even this very simple model can be used for predicting the occurrence of liquefaction if used in conjunction with the endochronic formulation.

\section{Conclusion}

In this research, an endochronic-based approach to the simulation of the behavior of saturated sand under undrained dynamic loading was introduced. This model was implemented into the finite element program, PISA, and its capability of predicting variations of excess pore water pressure in cyclic simple shear and centrifuge tests was evaluated. Results of the simulations showed that this model had a good capability of considering the contractive dilative behavior of saturated sand in predicting the variation of excess pore water pressure. Results showed that the application of the endochronic model coupled with the model of Manzari \& Dafalias (1997) led to the favorable predictions of the variation of excess pore water pressure and liquefaction. By using the endochronic model coupled with Mohr-Coulomb, the trend of increasing pore water pressure and forecasting of occurrence of liquefaction can be acceptably modeled. This ability is useful for forecasting the occurrence of liquefaction by using a simple constitutive law such as Mohr-Coulomb combined with the endochronic formulations.

\section{References}

1. Lopez-Querol, S. and Blazquez, R. "Liquefaction, and cyclic mobility model for saturated granular media", International Journal for Numerical and Analytical Methods in Geomechanics, 30(5), pp. 413-439 (2006).

2. Dafalias, Y.F. "Overview of constitutive model used in velacs", In Verification of Numerical Procedures for the Analysis of Soil Liquefaction Problems, 2, Rotterdam, Balkema (1993).

3. Valanis, K.C. "A theory of viscoplasticity without a yield surface. Part 1: General theory", Arch. Mech., 23, pp. 535-551 (1971).

4. Han, C.W and Yang, R.J. "Application of the improved endochronic theory of plasticity to loading with multiaxial strain-path", International Journal of Nonlinear Mechanics, 18(5), pp. 395-408 (1983).

5. Valanis, K.C. and Read, H.E. "A new endochronic plasticity model for soils", In: Soil MechanicsTransient and Cyclic Loads, Wiley, pp. 375-417 (1982).

6. Bazant Zdenek, P. and Krizek Raymond, J. "Endochronic constitutive law for liquefaction of sand", 
Journal of The Engineering Mechanics Division, 102(2), pp. 225-238 (1976).

7. Zienkiewicz, O.C., Chang, C.T., and Hinton, E. "Nonlinear seismic response and liquefaction", International Journal for Numerical and Analytical Methods in Geomechanics, 2(4), pp. 381-404 (1978).

8. Finn, W.L. and Bhatia, S.K. "Endochronic theory of sand liquefaction", In Proceedings of the 7th Word Conference on Earthquake Engineering, Turkey: Istanbul (1980).

9. Blazquez, R. and Lopez-Querol, S. "Generalized densification law for dry sand subjected to dynamic loading", Soil Dynamics and Earthquake Engineering, 26(9), pp. 888-898 (2006).

10. Cuellar, V. "Rearrangement measure theory applied to dynamic behavior of sand", PhD Thesis, Civil Engineering Department, Northwestern University, Evanston, IL, U.S.A. (1974).

11. Papadimitriou, A., Bouckovalas G., and Dafalias Y. "Plasticity model for sand under small and large cyclic strains", Journal of Geotechnical and Geoenvironmental Engineering, 127(11), pp. 973-983 (2001).

12. Blazquez, R. and Lopez-Querol, S. "Endochronicbased approach to the failure of the lower San Fernando dam in 1971", Journal of Geotechnical and Geoenvironmental Engineering, 133(9), pp. 1144-1153 (2007).

13. Lopez-Querol, S. and Blazquez, R. "Validation of a new endochronic liquefaction model for granular soil by using centrifuge test data", Soil Dynamics and Earthquake Engineering, 27(10), pp. 920-937 (2007).

14. Chan, D. and Morgenstern, N. "SAGE: a finite element program for stress analysis in geotechnical engineering", Technical report, Geotechnical Group. The University of Alberta (1998).

15. Chan, D. "PISA: Program for incremental stress analysis", User Manual, version 1.0, PISA Inc (1997).

16. Pak, A. "Numerical modelling of hydraulic fracturing", Ph.D. Thesis, Department of Civil and Environmental Engineering, University of Alberta (1997).

17. Shahir, H. "Dynamic analysis of saturated porous media for numerical simulation of liquefaction", M.Sc. Thesis, Department of Civil Engineering, Sharif University of Technology, Tehran, Iran (2001).

18. Rahmani, A., Ghasemi Fare, O., and Pak, A. "Investigation of the influence of permeability coefficient on the numerical modeling of the liquefaction phenomenon", Scientia Iranica : Transactions A, 19(2), pp. 179-187 (2012).

19. Shahir, H., Pak, A., Taibeat, M., and Jeremic, B. "Evaluation of variation of permeability in liquefiable soil under earthquake loading", Computers and Geotechnics, 40, pp. 74-88 (2012).

20. Biot, M.A. "General theory of three dimensional consolidation", Journal of Applied Physics, 12(2), pp. 155-168 (1941).
21. Zienkiewicz, O.C. and Shiomi, T. "Dynamic behavior of saturated porous media; the generalized biot formulation and its numerical solution", International Journal for Numerical Methods in Engineering, 8, pp. 71-96 (1984).

22. Katona, M.G. and Zienkiewicz, O.C. "A unified set of single step algorithms part 3: The beta-m method, a generalization of the newmark scheme", International Journal of Numerical Methods in Engineering, 21, pp. 1345-1359 (1985).

23. Arulanandan, K. and Sybico, J. "Post liquefaction settlement of sand", In Proceeding of the Worth Memorial Symposium England, Oxford University (1992).

24. Jafarzadeh, F. and Yanagisawa, E. "Settlement of sand models under unidirectional shaking", First International Conference on Earthquake Geotechnical Engineering, IS Tokyo (1995).

25. Shahir, H. and Pak, A. "Estimating liquefactioninduced settlement of shallow foundations by numerical approach", Computers and Geotechnics, 37(3), pp. 267-279 (2010).

26. Arulmoli, K., Muraleetharan, K.K., Hossain, M.M., and Fruth, L.S. "Velacs: Verification of liquefaction analyses by centrifuge studies-laboratory testing program", Soil Data Report, Earth Technology Corporation (1992).

27. Taboada, V.M. and Dobry, R. Experimental results of model no. 1 at RPI, University of California, California Institute of Technology (1993).

28. Manzari, M.T. and Dafalias, Y.F. "A critical state two-surface plasticity model for sands", Geotechnique, 47(2), pp. 255-272 (1997).

\section{Biographies}

Ali Pak received his BS degree in Civil Engineering in 1984, his MS degree in Soil and Foundation Engineering in 1989, and his $\mathrm{PhD}$ in Geotechnical Engineering from University of Alberta in 1997. Since then, he has been active in education and research at the Civil Engineering Department of Sharif University of Technology. Professor Pak has extensively published his works in high ranking journals and conferences worldwide. His research interests are soil liquefaction, soil improvement, environmental geotechnics, and petroleum geomechanics.

Mohammad Ali Iranmanesh received his BS degree in Civil Engineering in 2012, his MS degree in Soil and Foundation Engineering in 2014 and his $\mathrm{PhD}$ in Geotechnical Engineering at the Civil Engineering Department of Sharif University of Technology in 2020. His research interests are soil liquefaction, computational geomechanics, petroleum geomechanics, and soil improvement. 